\title{
Documentos
}

\section{Neoplasia vaginal intraepitelial}

\author{
Laura Baquedano M. ${ }^{1}$, Marta Lamarca B. ${ }^{1}$, Yasmina José G. ${ }^{1}$, Patricia Rubio C. ${ }^{1}$, \\ Miguel Ángel Ruiz C. 1
}

${ }^{1}$ Servicio de Ginecología, Hospital Universitario Miguel Servet. Zaragoza, España.

\section{RESUMEN}

Objetivo: Actualizar los conocimientos disponibles sobre la neoplasia vaginal intraepitelial (VAIN) especialmente en el diagnóstico y tratamiento. Métodos: Revisión de la literatura en Pubmed de los últimos 20 años, especialmente de los publicados desde 2005 hasta la actualidad y considerando sobre todo los ensayos clínicos aleatorizados. Resultados: Su prevalencia real es desconocida, aunque es una patología rara generalmente en mujeres posmenopaúsicas. Su fisiopatología es similar a la neoplasia cervical intraepitelial, con el HPV como principal factor de riesgo, sobre todo el serotipo 16, pero su progresión a cáncer es menor. Un grupo importante es el de VAIN tras histerectomía, que supone el $48-70 \%$ del total de las VAIN. La colposcopia para el diagnóstico no es sencilla y se aconseja preparación con estrógenos en las pacientes menopáusicas y utilización de ácido acético y lugol para identificar las zonas sospechosas. Las tres opciones terapéuticas son la cirugía (excisional, láser, ultrasonidos), braquiterapia y tratamiento médico (imiquimod, 5-fluorouracilo, ácido tricloroacético). Se expone la técnica y las ventajas e inconvenientes de cada uno de ellos. Conclusión: Aunque la VAIN es una entidad rara, es preciso tenerla en cuenta ante un diagnóstico de lesión citológica. Su diagnóstico a veces no es sencillo y el tratamiento dependerá del grado, la localización y el tamaño de la lesión y las circunstancias personales de la paciente. Se necesitan más ensayos aleatorizados que comparen la eficacia entre las distintas opciones terapéuticas y su repercusión en la calidad de vida de las pacientes.

\section{PALABRAS CLAVE: Neoplasia vaginal intraepitelial, colposcopia, histerectomía, láser, colpectomía, imiquimod}

\section{SUMMARY}

Aims: Update the knowledge about vaginal intraepithelial neoplasia (VAIN) with special emphasis on diagnosis and therapeutic management. Method: Electronic search of Pubmed of all kinds of articles about the VAIN, for the last 20 years with special attention to those published from 2005 to the present and considering especially randomized clinical trials. Results: Its prevalence is unknown, although it is a rare condition that usually occurs in postmenopausal women. The physiopathology is similar to cervical intraepithelial neoplasia, being HPV the main risk factor, particularly serotype 16, but its progression to cancer is lower. An important group is VAIN after hysterectomy, $48-70 \%$ of total ValN. Colposcopy for the diagnosis is not easy and in patients with postmenopausal the preparation with local estrogen is necessary and use both of acetic acid and lugol to identify suspicious areas. The three treatment options are surgery (excisional, laser, ultrasound), brachytherapy and medical management (imiquimod, 5-fluorouracil, tricholoroacetic acid). The technique and the advantages and disadvantages of each are explained. Conclusion: Although VAIN is rare, it must take it into account before a cytological diagnosis of dysplasia. Its diagnosis is sometimes not easy 
and the treatment depends on the extent, location and size of the lesion and the individual preferences of the patient. We need more randomized trials comparing different treatment options and also their impact on quality of life of patients.

\section{KEY WORDS: Vaginal intraepithelial neoplasia, colposcopy, hysterectomy, laser, colpectomy, imiquimod}

\section{INTRODUCCIÓN}

La neoplasia vaginal intraepitelial (VAIN) es una entidad rara, si bien al ser asintomática su prevalencia real es difícil de conocer. El empleo generalizado de la citología de cribado ha facilitado su detección, estimándose su prevalencia en 1 a $3 \%$ de las neoplasias intraepiteliales cervicales (CIN). La edad media al diagnóstico se sitúa en torno a los 50 años (1), pero con un rango de edad muy amplio, entre los 20 y 80 años.

Dada la rareza de la VAIN, hay pocos estudios publicados que permitan conocer bien su historia natural y su tasa real de progresión a cáncer y según las series actualmente se estima en torno al $9-10 \%$ en las pacientes no tratadas (2). En cualquier caso, la progresión de VAIN a cáncer de vagina es menor que de CIN a cáncer de cérvix.

\section{FISIOPATOLOGÍA Y FACTORES DE RIESGO}

Tiene rasgos histopatológicos similares a la CIN o la neoplasia vulvar intraepitelial (VIN) y con frecuencia se desarrolla como extensión de la $\mathrm{CIN}$, sobre todo en el tercio superior de la vagina. Comparte con ellas la fisiopatología y los factores de riesgo, incluyendo HPV, tabaquismo, conducta sexual e inmunosupresión, por lo que en muchas ocasiones se asocia a otra neoplasia intraepitelial del tracto genital inferior. El $75 \%$ de las pacientes con VAIN tienen de forma sincrónica o metacrónica lesiones intraepiteliales o invasivas de cérvix y vulva, constituyendo lo que se denomina como enfermedad multicéntrica del tracto genital inferior (TGI).

EI HPV se encuentra en el $94 \%$ de las VAIN (3) y el serotipo que con mayor frecuencia se detecta es el 16 (4). Un reciente estudio, caso control, en población china, determinó como factores de riesgo para la aparición de VAIN: estado menopáusico, histerectomía previa y como factores más potentes, la infección por HPV e historia previa de CIN o cáncer invasor de cérvix (5).

Las pacientes inmunodeprimidas con VAIN suelen ser más jóvenes y debido a la persistencia de HPV, por su estado inmunitario comprometido y al mantenimiento de los factores de riesgo, tienen tendencia a la recidiva.

VAIN tras histerectomía: Un grupo epidemiológico importante es el de las pacientes histerectomi- zadas, que representan $48-70 \%$ de las VAIN. Entre 15 y $39 \%$ según las series después de histerectomía por $\mathrm{CIN}$, no en relación con la extirpación incompleta del CIN, sino más bien por la persistencia de la infección por HPV y los factores de riesgo, o por una reinfección posterior. Se estima que la incidencia de VAIN después de una histerectomía por CIN en los 10 años siguientes a la cirugía es $0,91 \%(6)$, aunque también puede aparecer tras cirugía por procesos benignos. La aparición de la lesión suele ser tardía tras la cirugía, sin embargo las recomendaciones europeas para el seguimiento tras histerectomía por CIN aconsejan realizar únicamente dos tomas citológicas a los 6 y 18 meses, y si son negativas, no repetirlas (7).

VAIN tras radioterapia. Puede diagnosticarse tras la irradiación de la pelvis por un proceso maligno ginecológico. Estos casos parecen ser refractarios a los tratamientos y más propensos a la progresión a cáncer (8).

\section{CLÍNICA Y DIAGNÓSTICO}

Generalmente se trata de lesiones asintomáticas que se diagnostican dentro del programa de cribado del cáncer cervical (1), cuya localización más frecuente es el tercio superior de la vagina.

La exploración colposcópica vaginal no es sencilla debido al tejido vaginal redundante o la atrofia postmenopáusica (en este caso se recomienda un tratamiento previo con estrógenos locales las 3 semanas previas al procedimiento). Se aplica en primer lugar ácido acético al 3-5\%; las lesiones de bajo grado se muestran como epitelio acetoblanco con mayor frecuencia y en las de alto grado se pueden observar además cambios vasculares tipo punteado o mosaico. Seguidamente se aplicará lugol de forma generosa para localizar mejor las lesiones (Figura 1). Hay que tener en cuenta la multifocalidad del $50 \%$ de las VAIN. El diagnóstico será siempre anatomopatológico y para ello se biopsiarán las áreas sospechosas, teniendo en cuenta que en las situadas en el tercio inferior de la vagina suelen requerir la utilización de anestesia local. La hemostasia se logra con barritas de nitrato de plata o pasta de Monsel. Es preciso biopsiar todas las áreas sospechosas, especialmente las de la cúpula vaginal, donde puede encontrarse infiltración hasta en un $28 \%$ de las pacientes. 


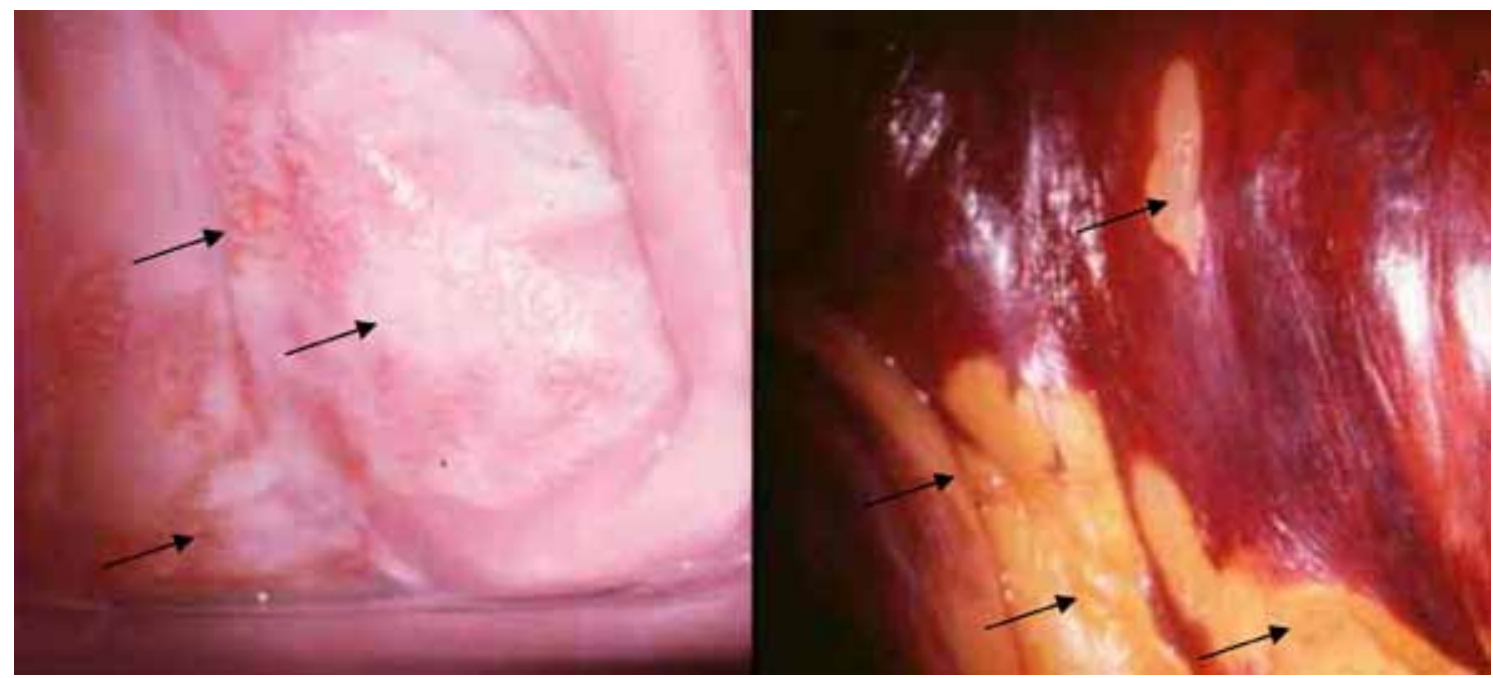

Figura 1. Cambios colposcópicos sugestivos de VAIN tras ácido acético y lugol (flechas).

\section{TRATAMIENTO}

No existe consenso nacional ni internacional sobre la conducta idónea para el tratamiento de la VAIN; en la literatura sólo existen estudios retrospectivos y series de casos. La lesión debe ser individualizada y el tratamiento dependerá de la extensión, grado, localización, edad, estado general, capacidad de realizar el seguimiento y siempre consensuar con la paciente, explicándole las opciones existentes. La multifocalidad y la asociación con el CIN son los factores más importantes para la recurrencia tras el tratamiento $(9,10)$. En el futuro la vacunación para el HPV será la llave para reducir la incidencia de la neoplasia intraepitelial del tracto genital inferior.

\section{Observación}

Se aconseja en lesiones de bajo grado, especialmente en mujeres jóvenes, con seguimiento cada 6 meses de las lesiones, que han de estar perfectamente documentadas y descritas para valorar su evolución. Se ha visto que las lesiones que no están asociadas a CIN ni VIN tienen mayor probabilidad de regresión ( $91 \%$ vs $67 \%$ ) (2).

\section{Cirugía}

Cirugía excisional. Permite excluir un cáncer invasivo y estudiar los márgenes. Sólo es necesario resecar la mucosa. No se ha demostrado ningún beneficio en el riesgo de recurrencia o de cáncer, si se extirpa también la fascia o el músculo, y sí una mayor tasa de complicaciones (11). Para ello, puede ser útil la inyección submucosa de un anestésico local con o sin adrenalina o suero salino, que ayudará a separar los planos. Puede realizarse con bisturí frío o mediante electrocirugía con asa de diatermia, teniendo cuidado en este caso de no producir lesiones térmicas en tejidos adyacentes, sobre todo vejiga y recto. Las tasas de éxito se sitúan entre el 66 y $83 \%(11,12,13)$. Se considera el tratamiento de elección en lesiones pequeñas y únicas, y en la VAIN tras histerectomía, donde el láser no tiene aplicación debido a que la lesión puede estar enterrada en la cicatriz de la cúpula vaginal y ocultar un carcinoma.

La colpectomía consiste en la eliminación de la mucosa vaginal, que podrá ser parcial o total, y ha demostrado las tasas más bajas de recurrencia (12\%) (14). Debido a las complicaciones por posible lesión de los órganos adyacentes y su impacto negativo en la sexualidad de la paciente, se reserva como segunda opción ante recidivas o enfermedad multifocal, sobre todo en mujeres mayores.

Láser. Puede utilizarse como una herramienta destructiva o excisional. El láser excisional es menos empleado, pero en una serie corta de casos proporciona mejores tasas de curación que el destructivo (15). El tratamiento destructivo de elección es la vaporización mediante láser $\mathrm{CO} 2$, que permite buenos resultados estéticos y funcionales. Deben biopsiarse previamente todas las lesiones para descartar infiltración. Las lesiones únicas se vaporizan con un margen de $1 \mathrm{~cm}$ y en las multifocales o muy extensas se vaporiza ampliamente toda el área afecta en una o dos sesiones. Según 
las series publicadas, el porcentaje de curación varía entre el $69-87,5 \%(13,15,16)$. Es la técnica de elección para las mujeres jóvenes o que no desean cirugía o en lesiones multifocales. No está indicada cuando la cúpula vaginal está implicada por su acceso limitado y el riesgo de daño de las estructuras vecinas.

Cirugía aspirativa ultrasónica. Consiste en la eliminación selectiva del tejido afecto mediante ultrasonido. Su tasa de curación está entre el 74 $80 \%(17,18)$. Un ensayo aleatorizado comparó el empleo de ultrasonido y la vaporización láser, encontrando similares tasas de recurrencia, pero con menor dolor postoperatorio en las pacientes tratadas con ultrasonido (19).

\section{Braquiterapia}

La radioterapia interna vaginal conlleva una morbilidad importante y debe reservarse para casos seleccionados. Además existe riesgo de desarrollar cáncer vaginal y por otra parte, su aplicación limita su uso en un futuro y también la cirugía, por la fibrosis que provoca. Por todo ello, la radioterapia no se recomienda como primera línea en el tratamiento de la VAIN y sólo debe considerarse en casos resistentes a los tratamientos convencionales.

\section{Tratamiento médico}

5-fluorouracilo. La aplicación local de crema al $5 \%$ una vez a la semana durante 10 semanas, ha demostrado altas tasas de curación aunque sus efectos secundarios (ardor, dispareunia, úlceras crónicas, disconfort vaginal) pueden limitar su aceptabilidad (20). No aprobada por la FDA para esta indicación.

Imiquimod. Fármaco inmunomodulador que estimula la actividad de las células del sistema inmunitario (natural Killer, células de Langerhans, macrófagos, linfocitos B) ha demostrado buenos resultados en series cortas para el tratamiento de lesiones de bajo grado con aplicación mediante guía colposcópica $(3,21)$.

Ácido tricoloacético. Su uso al $50 \%$ una vez a la semana durante 1 a 4 semanas se ha empleado para el tratamiento de las lesiones de bajo grado con remisión completa en todas las pacientes y leves efectos secundarios locales (22).

\section{CONCLUSIÓN}

La neoplasia vaginal intraepitelial es una entidad rara, cuya prevalencia real es difícil de conocer dado su carácter asintomático. Su fisiopatología y factores de riesgo son paralelos a su homónimo en el cérvix, aunque su progresión a cáncer invasor es menor. La localización más frecuente es el tercio superior de vagina, pero hay que tener en cuenta que en el $50 \%$ de los casos se trata de lesiones multifocales y la mayoría se asocian a otras lesiones del tracto genital inferior. El diagnóstico es anatomopatológico mediante biopsia dirigida por colposcopia de todas las lesiones sospechosas a la exploración, aunque en algunas ocasiones su identificación puede ser dificultosa. El manejo ha ser individualizado y dependerá de la extensión, grado, localización, edad, estado general, capacidad de realizar el seguimiento y siempre debe consensuarse con la paciente. Si se trata de lesiones de bajo grado, se recomendará la observación con un seguimiento adecuado. La escisión quirúrgica es el tratamiento de primera línea para lesiones únicas, pequeñas y en el VAIN tras histerectomía, mientras que el láser es la técnica de elección para las mujeres jóvenes o que no desean cirugía o en lesiones multifocales. Además se han descrito diferentes tratamientos médicos que han demostrado buenos resultados en series cortas de casos, por lo que en la actualidad se suelen reservar para una segunda línea terapéutica.

\section{REFERENCIAS}

1. Meler E, Olivella A, Alonso I, García S, Torné A,Ordi J, Puig Tintoré LM. The epidemiology and clinical characteristics of vaginal intraepithelial neoplasia (VAIN). J Low Genit Tract Dis 2007;11:198-9.

2. Aho M, Vesterinen E, Meyer B, Purola E, Paavonen J. Natural history of vaginal intraepithelial neoplasia. Cancer 1991;68:195-7.

3. Vuyst $\mathrm{H}$, Clifford GM, Nascimiento MC, Madeleine MM, Franceschi S. Prevalence and type distribution of human papillomavirus in carcinoma and intrepithelial neoplasia of the vulva, vagina and anus: a metaanalysis. Int J Cancer 2009;124:1626-36.

4. Smith JS, Backes DM, Hoots BE, Kurman RJ, Pimenta JM. Human papillomavirus type-distribution in vulvar and vaginal cancers and their associated precursors. Obstet Gynecol 2009;113:917-24.

5. Li H, Guo YL, Zhang JX, Qiao J, Geng L. Risk factors for the development of vaginal intraepithelial neoplasia. Chin Med J (Engl) 2012;125:1219-23.

6. Gemmell J, Holmes DM, Duncan ID. How frequently need vaginal smears be taken after hysterectomy for cervical intraepithelial neoplasia? Br J Obstet Gynaecol 1990;97:58-61.

7. David L, Simon L. Colposcopy and Programme Management: Guidelines for the NHS Cervical Screening Programme. 2nd ed. London, UK: NHS CSP; 2010. Publication No 20.

8. Liao JB, Jean S, Wilkinson-Ryan I, Ford AE, Tanyi JL, Hagemann AR, et al. Vaginal intraepithelial neoplasia (VAIN) after radiation therapy for gynecologic malignancies: a clinically recalcitrant entity. Gynecol Oncol 2011;120:108-12. 
9. Dodge JA, Eltabbakh GH, Mount SL, Walker RP, Morgan A. Clinical features and risk of recurrence among patients with vaginal intraepithelial neoplasia. Gynecol Oncol 2001;83:363-9.

10. Kondi-Pafiti A, Grigoriadis C, Kalampokas T, Filippidou A, Salakos N, Hassiakos D. Clinicopathological study of 112 cases of benign, pre-invasive and invasive lesions of the vagina: a 15-year review. Eur J Gynaecol Oncol 2012;33:463-6.

11. Curtis $P$, Shepherd JH, Lowe DG, Jobling T. The role of partial colpectomy in the management of persistent vaginal neoplasia after primary treatment. $\mathrm{Br} \mathrm{J}$ Obstet Gynaecol 1992;99:587-9.

12. Cheng D, Ng TY, Ngan HY, Wong LC. Wide local excision (WLE) for vaginal intraepithelial neoplasia (VAIN). Acta Obstet Gynecol Scand 1999;78:648-52.

13. Rome RM, England PG. Management of vaginal intraepithelial neoplasia: a series of 132 cases with longterm followup. Int J Gynecol Cancer 2000;10:382-90.

14. Indermaur MD, Martino MA, Fiorica JV, Roberts WS, Hoffman MS. Upper vaginectomy for the treatment of vaginal intraepithelial neoplasia. Am J Obstet Gynecol 2005;193:577-80.

15. Sideri M, Spinaci L, Spolti N, Schettino F. Evaluation of $\mathrm{CO} 2$ laser excision or vaporization for the treatment of vulvar intraepithelial neoplasia. Gynecol Oncol 1999;75:277-81.
16. Perrotta M, Marchitelli CE, Velazco AF, Tauscher $P$, Lopez G, Peremateu MS. Use of CO2 laser vaporization for the treatment of high-grade vaginal intraepithelial neoplasia. J Low Genit Tract Dis 2013;17:23-7.

17. Matsuo K, Chi DS, Walker LD, Rosenshein NB, Im DD. Ultrasonic surgical aspiration for vaginal intraepithelial neoplasia. Int J Gynaecol Obstet 2009;105:71-3.

18. Robinson JB, Sun CC, Bodurka-Bevers D, Im DD, Rosenshein NB. Cavitational ultrasonic surgical aspiration for the treatment of vaginal intraepithelial neoplasia. Gynecol Oncol 2000;78:235-41.

19. Von Gruenigen VE, Gibbons HE, Gibbins K, Jenison EL, Hopkins MP. Surgical treatments for vulvar and vaginal dysplasia: a randomized controlled trial. Obstet Gynecol 2007;109:942-7.

20. Gonzalez Sanchez JL, Flores Murrieta G, Chavez Brambila J, Deolarte Manzano JM, Andrade Manzano AF. Topical 5-fluorouracil for treatment of vaginal intraepithelial neoplasms [in Spanish]. Ginecol Obstet Mex 2002;70:244-7.

21. lavazzo C, Pitsouni E, Athanasiou S, Falagas ME. Imiquimod for treatment of vulvar and vaginal intraepithelial neoplasia. Int J Gynaecol Obstet 2008;101:3-10.

22. Lin H, Huang EY, Chang HY, ChangChien CC. Therapeutic effect of topical applications of trichloroacetic acid for vaginal intraepithelial neoplasia after hysterectomy. Jpn J Clin Oncol 2005;35:651-4. 